\section{MPV may reflect subcinical platelet activation in diabetic patients with and without diabetic retinopathy}

M Citirik ${ }^{1}$, E Beyazyildiz ${ }^{1}$, M Simsek $^{1}$ O Beyazyildiz ${ }^{1}$ and IC Haznedaroglu ${ }^{2}$
${ }^{1}$ SB Ankara Ulucanlar Eye Education and Research Hospital, Ankara, Turkey

\section{${ }^{2}$ Department of} Hematology, Faculty of Medicine, Hacettepe University, Ankara, Turkey

Correspondence: M Citirik, Fakulteler Mah. SB Ankara Ulucanlar Eye Education, Yazgan Sk. No: 34/12, Cebeci, 06590 Ankara, Turkey Tel: +90 3123126261 ; Fax: +903123124827 . E-mail: mcitirik@ hotmail.com

Received: 25 July 2014 Accepted in revised form: 6 October 2014 Published online: 19 December 2014

\begin{abstract}
Purpose To search subclinical platelet activation via detecting three important platelet activation parameters; mean platelet volume (MPV), platelet distribution width (PDW), and plateletcrit (PCT) in diabetic retinopathy (DR) in comparison with those in healthy adults as controls.

Methods This prospective study included 140 patients who were followed-up at the Ankara Ulucanlar Eye Education and Research Hospital, and 40 normal subjects. All patients and control subjects underwent complete ophthalmologic evaluation. Of patients with type 2 diabetes, 43 cases with diabetes mellitus (DM) have no DR (Group 1), 45 cases with DM have nonproliferative DR (NPDR) (Group 2), and 52 cases with DM have proliferative DR (PDR) (Group 3). In addition, 40 age- and sex-matched healthy controls (Group 4) were included into the study. MPV, PDW, and PCT were measured in the studied groups.

Results The MPV levels were significantly altered in Group 1, Group 2, and Group 3 patients when compared with those in the controls $(P<0.05)$, whereas PDW and PCT levels were not significantly changed among groups $(P>0.05)$.

Conclusion The data provided a significant association between MPV levels and DM. Diabetic patients have increased MPV values compared with healthy subjects, but MPV levels were not altered together with the DR stage. Diabetic and DR patients have no different PDW and PCT values compared with healthy subjects. MPV may be a clue for the reflection of subclinical platelet activation in DM regardless of the DR stage.
\end{abstract}

Eye (2015) 29, 376-379; doi:10.1038/eye.2014.298; published online 19 December 2014

\section{Introduction}

Diabetic retinopathy (DR) is the most common and specific microangiopathy of diabetes mellitus (DM). ${ }^{1}$ There are clearly defined risk factors for DR, such as hyperglycemia, hypertension, dyslipidemia, and diabetes duration. ${ }^{2}$ It has also shown that functional and structural changes in retinal arterioles are also a risk factor for $\mathrm{DR}^{3}$ Abnormal insulin activation in patients with diabetes leads to an increased platelet function and then microvascular complications. ${ }^{4}$

Mean platelet volume (MPV) reflects the average size of platelets present in a person's blood sample. ${ }^{5}$ It is a marker indicating subclinical platelet activation and is an independent risk factor for various vascular episodes such as acute myocardial infarction, ${ }^{5}$ coronary artery disease, ${ }^{6}$ cerebral ischemia, ${ }^{7}$ and peripheral artery disease. ${ }^{8}$ There are limited reports in literature showing relation between DR and platelet indices. Increased MPV has been found to be associated with increased risk of retinopathy in diabetic patients. ${ }^{9}$ Besides $\mathrm{MPV}$, other platelet indices platelet distribution width (PDW) and plateletcrit (PCT) may have an important role on vascular events, such as atherosclerosis and thrombosis. ${ }^{10}$ PDW reflects how uniform the platelets are in size. PCT is a measure of total platelet mass. ${ }^{10}$

The aim of this study is to assess possible relation of DR with platelet indices MPV, PDW, and PCT. To the best of our knowledge, this is the first study that describes relation between 
the markers of subclinical platelet activation including the PCT, PDW, and DR.

\section{Patients and methods}

We prospectively evaluated 140 patients with type 2 DM between August 2012 and December 2013. All of the study procedures were conducted in accordance with the Declaration of Helsinki, and informed consents were taken from all of the participants after approval from the Institutional Review Board. This study was approved by The Ethical Committee of Diskapi Training and Research Hospital. All patients were Turkish Caucasians. The presence of DM in all patients had been confirmed by the corresponding internal medicine department. The status of retinopathy was also assessed by fundus photography, fluorescein angiography, and optical coherence tomography. Early Treatment of Diabetic Retinopathy Study criteria were used to define various stages of DR. ${ }^{11}$

The cases were separated into four Groups based on the findings of clinical ocular examination. The first Group included 43 diabetic patients having no DR (Group 1); the second Group included 45 diabetic patients having nonproliferative DR (NPDR) (Group 2); the third Group included 52 diabetic patients having proliferative DR (PDR) (Group 3); the fourth Group included 40 age- and sex-matched healthy adult subjects (Group 4) serving as controls with no history of systemic or ocular diseases. The diseases/conditions and drugs that are known to be associated with changes in platelet function were excluded from the study. In addition, patients and healthy volunteers who had smoking and drinking habits, or who had history of hypertension and dyslipidemia were not included in the study. To avoid the effect of diurnal variation in the hemostatic system, all sampling procedures were performed in a fasting state and in the morning state. Blood was collected with minimal stasis in EDTA vacutainer tubes and studied within $60 \mathrm{~min}$.

Differences among the four Groups for platelet parameters were evaluated using one-way ANOVA, where applicable. Bonferroni test was used as post hoc test after one-way ANOVA. The level of significance was set at $<0.05$. All statistical analyses of the study were performed by using SPSS for Windows (SPSS Inc., Chicago, IL, USA) software.

\section{Results}

The clinical research was performed on 180 cases. Of these patients, 96 women (53.3\%) and 84 men $(46.7 \%)$ were available for the evaluation. The average age of the patients was (mean \pm SD) $60.39 \pm 8.52$ in Group 1, $61.44 \pm 9.31$ in Group 2, $59.40 \pm 7.17$ in Group 3, and
$58.82 \pm 7.91$ in Group 4. The time elapsed since the diagnosis of DM was $9.3 \pm 2.5$ years for Group 1, $11.2 \pm 2.7$ years for Group 2, and $13.8 \pm 3.9$ years for Group 3. There was no difference regarding the age and gender of the patients in the three studied Groups $(P>0.05)$ (Table 1$)$.

The blood samples showed a marked elevation in MPV levels in our diabetic patients having no DR $(P=0.01)$, NPDR $(P=0.01)$, and PDR $(P=0.01)$ patients, compared with controls. The mean serum level of MPV was $7.94 \pm 0.63$ in Group 1, $8.05 \pm 0.76$ in Group 2, $8.10 \pm 0.68$ in Group 3, and $7.74 \pm 0.78$ in Group 4 (Table 2). However, there was no significant difference of mean MPV values among DR stages $(P>0.05)$.

Mean PDW values were 14.85 \pm 1.27 in Group 1, $15.15 \pm 1.19$ in Group 2, $14.92 \pm 1.15$ in Group 3, and $14.64 \pm 1.16$ in Group 4 (Table 2). There was no statistically significant difference of PDW values among groups $(P>0.05)$. There was no significant difference between diabetic groups and healthy subjects regarding PCT levels $(P>0.05)$. There was also no significant difference of mean PDW values among DR stages $(P>0.05)$.

Mean PCT values were 0.20 \pm 0.05 in Group 1, $0.20 \pm 0.06$ in Group 2, $0.19 \pm 0.04$ in Group 3, and $0.19 \pm 0.04$ in Group 4 (Table 2). There was no statistically significant difference of PCT values among groups $(P>0.05)$. There was no significant difference between diabetic groups and healthy subjects regarding PCT levels $(P>0.05)$. There was also no significant difference of mean PCT values among DR stages $(P>0.05)$.

\section{Discussion}

DR, the most common retinal vascular disease, is the leading cause of new blindness in adults during the third through sixth decade of life. ${ }^{1}$ The pathogenesis of diabetes includes prothrombotic state and increased thrombocyte aggregation caused by multiple different mechanisms. ${ }^{1,2}$ Diabetic patients have higher thrombocyte count and enhanced MPV, PDW values, and platelet activation can lead to the generation of vascular diseases. ${ }^{3,4}$ High MPV value means a large thrombocyte size. Large thrombocytes are younger, denser, and more active. Therefore higher MPV increases the chance of vascular complications. ${ }^{12,13}$ Previous studies disclosed that MPV was linked with coronary artery disease, myocardial infarction, peripheral artery disease, and cerebral ischemia. ${ }^{5-8}$ Several studies have showed that there were aggregates of thyrombocytes in retinal capillaries of diabetic rats. ${ }^{14,15}$ Tuzcu et al ${ }^{9}$ conducted an experiment with 192 patients and showed a correlation between MPV levels and DR. They showed that occurrence of DR increases with increasing MPV levels. 
Table 1 Demographic data of the groups

\begin{tabular}{lcccrr}
\hline & Group 1 $(\mathrm{n}=43)$ & Group 2 $(\mathrm{n}=45)$ & Group 3 $(\mathrm{n}=52)$ & Group 4 $(\mathrm{n}=40)$ & P-value \\
\hline Age (years) & $60.39 \pm 8.52$ & $61.44 \pm 9.31$ & $59.40 \pm 7.17$ & $58.82 \pm 7.91$ & 0.067 \\
Sex (male/female) & $22 / 21$ & $17 / 28$ & $20 / 32$ & $25 / 15$ & 0.296 \\
\hline
\end{tabular}

Table 2 The mean values of the MPV, PCT, and PDW and the comparison of platelet parameters (mean \pm SD) for four groups

\begin{tabular}{lrrrrr}
\hline & \multicolumn{1}{c}{ Group 1 } & Group 2 & Group 3 & Group 4 & P-value \\
\hline MPV & $7.94 \pm 0.63$ & $8.05 \pm 0.76$ & $8.10 \pm 0.68$ & $7.74 \pm 0.78$ & 0.01 \\
PCT & $0.20 \pm 0.05$ & $0.20 \pm 0.06$ & $0.19 \pm 0.04$ & $0.19 \pm 0.05$ & 0.48 \\
PDW & $14.85 \pm 1.27$ & $15.15 \pm 1.19$ & $14.92 \pm 1.15$ & $14.64 \pm 1.16$ & 0.27 \\
\hline
\end{tabular}

Abbreviations: MPV, mean platelet volume; PCT, plateletcrit; PDW, platelet distribution width.

They also showed that a 1.40-fold increase in the risk of retinopathy development and a 1.46-fold increase in the risk of proliferative DR as the MPV value increased. Similar studies have shown that DR has higher MPV values compared with healthy humans. ${ }^{12,16}$ Papanas et al ${ }^{17}$ have found higher MPV levels in diabetes patients in their study conducted on 416 subjects. They also showed that patients with DR have higher MPV levels compared with other diabetic patients. Moreover, they found that diabetic patients with microalbuminuria (indicates microvascular damage) have higher MPV levels than the nondiabetic group. Our study shows that diabetic patients have significantly higher MPV values compared with the healthy subjects. However, MPV levels were not altered along with the DR stage in the present study.

There is no standard way of measuring MPV and different type of measures can have differences up to $40 \%$ difference. ${ }^{18}$ Therefore to study MPV levels in DR patients, MPV measuring needs to be standardized. Lance et al showed elapsed time is very important in MPV measuring. They showed that optimal measurement duration should be $120 \mathrm{~min}$ after venipuncture. Thus, in our study the blood was collected and studied within $60 \mathrm{~min}$.

MPV and PDW are increase during platelet activation. PDW is a more specific marker of platelet activation as it does not increase during simple platelet swelling. ${ }^{19}$ Karagoz et al ${ }^{20}$ conducted a retrospective study with 110 lung cancer patients and they found that patients have higher PDW and platelet levels compared with healthy humans. They showed that thrombocytes which contain increased number pseudopodias with different sizes were an indicator of changing PDW levels. ${ }^{20}$ Vagdatli et $a l^{19}$ showed that PDW was a more specific marker for platelet activation. Jindal et $a l^{21}$ showed that there is a significant increase in PDW levels for diabetic patients and the increase is more significant in patients with microvascular complications. In our study, we found that diabetic patients have higher PDW levels compared with the control group, but the differences were not reached to statistically significant level. Similarly, DR patients have different PDW levels according to DR stages, but the difference was not statistically significant.

The interrelationships between PCT and DR had not been studied previously. PCT was accepted as an indicator of circulating platelets in a unit volume of blood. PCT is an effective screening tool for detecting platelet quantitative abnormalities, and it can be calculated as platelet $\times \mathrm{MPV}$ / $10^{7} .^{22}$ Akpinar et $a l^{23}$ determined that PCT has an important predictive value for Saphenous vein graft disease, and they emphasized that it could be used as a marker for antiplatelet therapy to prevent graft atherosclerosis in patients undergoing by-pass surgery. In present study, there was no significant difference between diabetic groups and healthy subjects regarding PCT levels. In addition, there was also no significant difference of mean PCT values in between DR stages.

There are several limitations in our present study. First, the number of patients is limited. Second, the design of this study is not convenient to investigate other potential risk factors in the development of thrombosis as the control group had healthy subjects with no history of systemic or ocular diseases.

The results of our present study show that diabetic patients have significantly higher MPV values than nondiabetic patients. Moreover, a gradual increase in MPV values was observed with increasing severity of DR. However, there is no statistically significant difference in terms of PCT and PDW values between the stages of DR. The results may be important for the vascular management of diabetic patients in everyday clinical practice as those platelet parameters are routinely detected in the complete blood count analyses. 


\section{Summary}

\section{What was known before}

- Three important platelet activation parameters were searched in DR and in healthy adult subjects.

\section{What this study adds}

- The MPV levels were significantly altered in patients when compared to those in the controls, whereas PDW and PCT levels were not significantly changed among groups.

\section{Conflict of interest}

The authors declare no conflict of interest.

\section{References}

1 Mohamed Q, Gillies MC, Wong TY. Management of diabetic retinopathy: a systematic rewiev. JAMA 2007; 298: 902-916.

2 Cheung N, Mitchell A, Wong TY. Diabetic retinopathy. Lancet 2010; 376: 124-136.

3 Curtis TM, Gardiner TA, Stitt AW. Microvascular lesions of diabetic retinopathy: clues towards understanding pathogenesis? Eye 2009; 23: 1496-1508

4 Vinik AM, Macagni A. Platelet dysfunction in type 2 diabetes. Diabetes Care 2001; 24: 1476-1485.

5 Khandekar MM, Khurana AS, Deshmukh SD, Kakrani AL, Katdare AD, Inamdar AK. Platelet volume indices in patients with coronary artery disease and acute myocardial infarction: an Indian scenario. J Clin Pathol 2006; 59: 146-149.

6 Tavil Y, Sen N, Yazici H, Turfan M, Hizal F, Cengel A et al. Coronary heart disease is associated with mean platelet volume in type 2 diabetic patients. Platelets 2010; 21: 368-372.

7 Pikija S, Cvetko D, Hajduk M, Trkulja V. Higher mean platelet volume determined shortly after the symptom onset in acute ischemic stroke patients is associated with a larger infarct volume on CT brain scans and with worse clinical outcome. Clin Neurol Neurosurg 2009; 111: 568-573.

8 Berger JS, Eraso LH, Xie D, Sha D, Mohler 3rd ER. Mean platelet volume and prevalence of peripheral artery disease, the National Health and Nutrition Examination Survey, 1999-2004. Atherosclerosis 2010; 213: 586-591.

9 Ayhan Tuzcu E, Arıca S, Ilhan N, Daglioglu M, Coskun M, Ilhan $\mathrm{O}$ et al. Relationship between mean platelet volume and retinopathy in patients with type 2 diabetes mellitus. Graefes Arch Clin Exp Ophthalmol 2014; 252: 237-240.

10 Davi G, Patrono C. Platelet activation and atherothrombosis. N Engl J Med 2007; 357: 2482-2494.

11 Early Treatment Diabetic Retinopathy Study design and baseline patient characteristics. ETDRS report number 7. Ophthalmology 1991; 98: 741-756.

12 Ates O, Kiki I, Bilen H, Keles M, Kocer I, Kulacoglu DN et al. Association of mean platelet volume with the degree of retinopathy in patients with diabetes mellitus. Eur J Gen Med 2009; 6: 99-102.

13 Kodiatte TA, Manikyam UK, Rao SB, Jagadish TM, Reddy M, Lingaiah HK et al. Mean platelet volume in type 2 diabetes mellitus. J Lab Physicians 2012; 4: 5-9.

14 Sima AA, Garcia-Salinas R, Basu PK. The BB Wistar rat: an experimental model for the study of diabetic retinopathy. Metabolism 1983; 32: 136-140.

15 Ishibashi T, Tanaka K, Taniguchi Y. Platelet aggregation and coagulation in the pathogenesis of diabetic retinopathy in rats. Diabetes 1981; 30: 601-606.

16 Zhong ZL, Han M, Chen S. Risk factors associated with retinal neovascularization of diabetic retinopathy in type 2 diabetes mellitus. Int J Ophthalmol 2011; 4: 182-185.

17 Papanas N, Symeonidis G, Maltezos E, Mavridis G, Karavageli E, Vosnakidis T et al. Mean platelet volume in patients with type 2 diabetes mellitus. Platelets 2004; 15: 475-478.

18 Beyan C, Beyan E. Letter to the editor: Mean platelet volume and diabetic retinopathy. Graefes Arch Clin Exp Ophthalmol 2013; 251: 2839-2840.

19 Vagdatli E, Gounari E, Lazaridou E, Katsibourlia E, Tsikopoulou F, Labrianou I. Platelet distribution width: a simple, practical and specific marker of activation of coagulation. Hippokratia 2010; 14: 28-32.

20 Karagoz B, Alacacioglu A, Bilgi O, Demirci E, Ozgun H, Erikci AA et al. Platelet count and platelet distribution width increase in lung cancer patients. Anatol J Clin Investig 2009; 3: 32-34

21 Jindal S, Gupta S, Gupta R et al. Platelet indices in diabetes mellitus: indicators of diabetic microvascular complications. Hematology 2011; 16: 86-89.

22 Bain BJ, Bates I. Basic haematological techniques. In: Lewis SM, Bain BJ, Bates I (eds). Dacie and Lewis Practical Haematology, 9th edn. Churchill Livingstone: Edinburgh, 2001, pp 19-46.

23 Akpinar I, Sayin MR, Gursoy YC, Karabag T, Kucuk E, Buyukuysal MC et al. Plateletcrit. A platelet marker associated with saphenous vein graft disease. Herz 2014; 39(1): 142-148 